\title{
The whole is greater than the sum
}

\author{
Stephen B Hanauer
}

This issue of Nature Clinical Practice Gastroenterology \& Hepatology emphasizes the inter-relationships between the gut, liver, brain and immune system pertaining to human diseases. The brain-gut connection has long been recognized from the perspective of the 'stress response' and is currently being used and explored for the treatment of both irritable bowel syndrome (IBS) and obesity. In addition, it has recently been recognized that visceral adipose tissue has both metabolic and immunoinflammatory properties.

In their Review, Filippo Cremonini and Nicholas $\mathrm{J}$ Talley discuss treatments targeting the putative mechanisms involved in the heterogeneous syndrome of IBS, with respect to physiologic models-modulation of gastrointestinal sensorimotor function, central processing of afferent information and neurohormonal stress responses-and the potential impact of microbial flora and local inflammation. They emphasize the spectrum of potential mechanisms that lead to individual IBS phenotypes and respective therapeutic targets. They conclude that, at present, these individual targets offer hope for "...improved long-term management of IBS" but that "disease modification rather than just symptom-based treatments must remain the goal". Unfortunately, disease modification, in the absence of specific genetic, physiologic, pathologic or biologic markers for disease subtypes will remain elusive. Nevertheless, the relationships between the central nervous system, enteric nervous system (including the neuroendocrine components), mucosal immune system, and luminal environment remain complex and are probably influenced by genetics, intestinal microflora and learned or emotional factors.
As a whole, the gastrointestinal tract provides more than 'just" a digestive function

SB Hanauer is Editorin-Chief of Nature Clinical Practice Gastroenterology \& Hepatology.

\section{Competing interests}

The author declared

competing interests; go to

the article online for details.

www.nature.com/clinicalpractice doi:10.1038/ncpgasthep0098
Similarly, George A Bray's Review on potential targets for appetite suppressants emphasizes the complex relationships between enteric and central neurochemical transmitters that contribute to the modulation of feeding "...from meal to meal and over time". These appetite suppressants include centrally acting sympathomimetic drugs, drugs acting on 5-hydroxytryptamine (serotonin), $\gamma$-aminobutyricacid receptors or cannabinoid receptors, as well as peptides that affect food intake. The peptides, which include leptin, cholecystokinin and ghrelin, are derived from gut sources (visceral adipose tissue) and impact on the hypothalamic intracellular signaling system.

Andreas Schäffler, Jürgen Schölmerich and Christa Büchler expand on the traditional view of the gastrointestinal system to include the visceral adipose tissue and it's emerging role, not only in intestinal and mesenteric diseases but also in "...regulating energy homeostasis, neuroendocrine, autonomic, immune, hematologic, angiogenic, vascular and endothelial functions". Clearly, the role of visceral adipose tissue requires further elucidation regarding the pathophysiologic effects of central obesity (hepatic steatosis, release of proinflammatory cytokines, and so on), as well as the local impact on Crohn's disease and uncommon disorders of the mesentery (panniculitis).

As a whole, the gastrointestinal tract provides more than 'just' a digestive function. The gut is also more than a 'second brain', with its impact on energy homeostasis, immunity, local and systemic inflammation. The effects on these systems in turn impact on a broad range of human diseases, not to mention the significant pleasures and displeasures that are attributed to appropriate and inappropriate functions of the gastrointestinal tract. 